\title{
Martingales via statistical convergence
}

\author{
Danjela Braho', ${ }^{1}$ Agron Tato ${ }^{2}$
}

${ }^{1}$ Department of Mathematics, Informatics and Physics, University "F. S. Noli", Korce, Albania

${ }^{2}$ Department of Mathematical Engineering, Polytechnic University of Tirana, Tirana, Albania

\section{ABSTRACT.}

In this paper martingales of statistical Bochner integrable functions with values in a Banach space are treated. In particular we have arrived some results for martingales and backwards martingales.

\section{Keywords}

Martingale; statistical convergence; statistical integrable

\section{SUBJECT CLASSIFICATION}

\author{
28B05;40A30
}

\section{INTRODUCTION}

The study of probability theory in abstract spaces became possible with the introduction of integration theories in such spaces. It plays a very important and useful role in the study of stochastic processes.

Continuously studied since its introduction, martingale theory is one of the central components of probability theory. Much of the work on Banach spaces done in the 1930's resulted from investigating how much of real variable theory might be extended to functions taking values in such spaces. In the 1960's, the theory of martingales of real or complex random variables has been extended by various authors to random variables taking values in a Banach space.

On the other hand one of the most recent generalizations of the concept of classical convergence of sequences,statistical convergence which has been introduced by Fast [13], who also introduced concepts such asLacunary statistical convergence and $\lambda$-statistical convergence, has become an active area of research under the name of statistical convergence since 1990s of the last century. It has appeared in a wide variety of topics such as [1], [3], [5], [8], [10], [12], [14], [15].

In [17] we have extended in case of statistical convergence some result present in [4] for martingales of statistical Bochnerintegrable functions on Banach space and in [16] theorems concerning the Radon-Nikodym property for vector valued martingales of statistically Bochnerintegrable functions are treated.

Also in this paper we treat convergence theorems for martingales of statistical Bochnerintegrable functions. Section 2 is devoted to listing various definitions and notations which we shall have to refer to later. In section 3 we prove some convergence theorems for martingales of statistical Bochnerintegrable variables.

\section{DEFINITIONS AND NOTATIONS}

In this section we recall some basic definitions and notations which form the background of the present work.

Let $E$ be a Banach space with norm \|. $\|, B(E)$ its unit ball and $E^{*}$ its dual. A subset $T$ of $E^{*}$ is called a total set over $E$, if $f(x)=0$ for each $f \in T$ implies $\mathrm{x}=0$. Throughout this note $(\Omega, \mathcal{F}, \mu)$ is a probability space and $\left(\mathcal{F}_{n}\right)_{n \in N}$ a family of sub- $\sigma$-algebras of $\mathcal{F}$ such that $\mathcal{F}_{m} \subset \mathcal{F}_{n}$ if $m<n$. Moreover, without loss of generality, we will assume that $\mathcal{F}$ is the completion of $\sigma\left(\bigcup_{n} \mathcal{F}_{n}\right)$.

Definition 1: A process $\left(f_{n}, B_{n}, n \in \square\right)$ is called a martingale if

$$
\left(f_{n}: n \geq 0\right) \text { is adapted to the filtration }\left\{B_{n}: n \geq 0\right\} \text {. }
$$

iii) $\forall n \leq n+1 \quad \forall A \in B_{n} \quad \int_{A} f_{n} d \mu=\int_{A} f_{n+1} d \mu$

We follow the notions about the statistical convergence of sequences introduced by Fridy [6], [7] and Gökhan and Güngör [9], as well as the approach of Schoenberg [11] about integration. The base line concept is the statistical convergence of Fridy [5]. 
A subset $\mathrm{A}$ of the ordered set $\mathrm{N}$ of natural numbers is said to have density $\delta(A)$, if $\lim \frac{|A(n)|}{n}=\delta(A)$, where $A(n)=\{k<n: k \in A\}$ and $|A|$ denotes the cardinality of set $A \subset N$. It is clearly that finite sets have the density zero and $\delta\left(\mathrm{A}^{\prime}\right)=1-\delta(A)$ if $\mathrm{A}^{\prime}=\mathrm{N} \backslash \mathrm{A}$. If a property $\mathrm{P}(\mathrm{k})=\{\mathrm{k} ; \mathrm{k} \in \mathrm{A}\}$ holds for all $k \in A$ with $\delta(\mathrm{A})=1$, we say that property $\mathrm{P}$ holds for all $\mathrm{k}$ that is a.a.k.

The vectorial sequence $\mathrm{x}$ is statistically convergent to the vector $\mathrm{L}$ of a vectorial normed space if for each $\varepsilon>0$

$\lim _{n \rightarrow \infty} \frac{1}{n}\left|\left\{k \leq n:\left\|\mathrm{x}_{k}-L\right\| \geq \varepsilon\right\}\right|=0$

i.e. $\left\|x_{k}-L\right\|<\varepsilon$ a.a.k. We write $s t-\lim x_{k}=L$.

The sequence $\mathrm{x}$ is statistically Cauchyif for each $\varepsilon>0$, there exists a number $N=N(\varepsilon)$ such that for $\mathrm{n}>\mathrm{N}(\varepsilon)$ $\left\|x_{k}-x_{N}\right\|<\varepsilon$.

Lemma Salat. A sequence $\left(x_{k}\right)$ is statistically convergent to $\mathrm{L}$ if and only if there exists a set $\mathrm{K}=\left\{\mathrm{k}_{1}<\mathrm{k}_{2}<\ldots\right\} \subset \mathrm{N}$ that $\delta(\mathrm{K})=1$ and $\lim _{n \rightarrow \infty}\left(x_{k_{n}}\right)=L$.

The set $\mathrm{K}$ is directed and the sequence $\left(x_{k_{n}}\right)$ is called the essential subsequence of $\left(x_{k}\right)$. The above lemma can be formulated:

A sequence $\left(x_{k}\right)$ is statistically convergent to $L$ if and only if there exists an essential subsequence $\left(x_{k_{n}}\right)$ which converges in usual meaning to limes L. We write $\lim x_{k}=L$.

We can formulate an immediate corollary of Salat's lemma.

The sequence $\left\{f_{k}(x)\right\}$ where $f_{k}: S \rightarrow X,(X$ a vectorial normed space) is statistically convergent to $f(x)$, if and only if, there exists an essential subsequence $\left(f_{k_{n}}\right)$ of it that is convergent to $f(x)$.

We follow the notions about the statistical convergence of sequences of functions from [9], [2]. Let $\left\{f_{k}\right\}$ be a sequence of functions with value in vectorial space. For each $x$ of the domain, we consider the functional sequence $\left(f_{k}(x)\right)$. We denote with $S$ the set of $x$ where the sequence $\left(f_{k}(x)\right)$ converges. The function $f$ defined as

$$
f(x)=\lim _{k \rightarrow \infty} f_{k}(x) ; x \in S
$$

is called the limit function of the sequence $\left\{f_{k}\right\}$, we say that sequence $\left\{f_{k}\right\}$ converges pointwise to $f$ for every $x \in S$.

Definition 2: A sequence of functions $\left\{f_{k}(x)\right\}$ is said to be pointwise statistically convergent to $f$ if for every $\varepsilon>0$ and $x \in S$,

$$
\left\|f_{k}(x)-f(x)\right\|<\varepsilon \text {. a.a. k. }
$$

We write $s t-\lim f_{k}(x)=f(x)$ on S.

Definition 3: A sequence of functions $\left\{f_{k}(x)\right\}$ is statistically Cauchyif for each $\varepsilon>0$, there exists a number $N=N(\varepsilon)$ such that for $n>N(\varepsilon)$

$$
\left\|f_{k}(x)-f_{N}(x)\right\|<\varepsilon
$$


Let $\mathrm{X}$ be a Banach space and $(\Omega, \Sigma, \mu)$ is a finite measure space and let $\left\{B_{n}, n \in \square\right\}$ be a filtration of $\Sigma$ and let $\left(f_{n}: n \geq 0\right)$ be a sequence of variables.

Definition 4. The function $f: S \rightarrow X$ is called statistically strong measurable by $\mu$ on $\mathrm{S}$ if every $\delta>0$ and every $\varepsilon>0$ there exists a integer $N(\varepsilon, \delta)$ such

$$
\lim _{n \rightarrow \infty} \frac{1}{n}\left|\left\{k \leq n:\left\|f_{k}(s)-f(s)\right\| \geq \varepsilon, \forall s \in S\right\}\right|=0
$$

for $k>N(\varepsilon, \delta)$ almost for every $s \in S$. In this case it is said that the sequence $f_{k}(\mathrm{~s})$ converges statistically strong almost everywhere uniformly by $\mu$ to the function $f$ on $S$.

Definition 5.[2] A $\mu-$ measurable function $f: \Omega \rightarrow X$ is called statistical Bochnerintegrable if there exists a sequence of simple functions $\left(f_{n}\right)$ such that

$$
s t-\lim _{n} \int_{\Omega}\left\|f_{n}-f\right\| d \mu=0
$$

In this case, st- $\int_{E} f d \mu$ is defined for each $E \in \sum$ by $\lim _{K} \underset{\mathrm{W}}{\mathbf{O}}\left\|f_{n}-f\right\| d m=0$.

The set of statistical Bochnerintegrable functions is a linear space we denote with $L^{\prime}{ }_{p}(m, \mathrm{X})$.

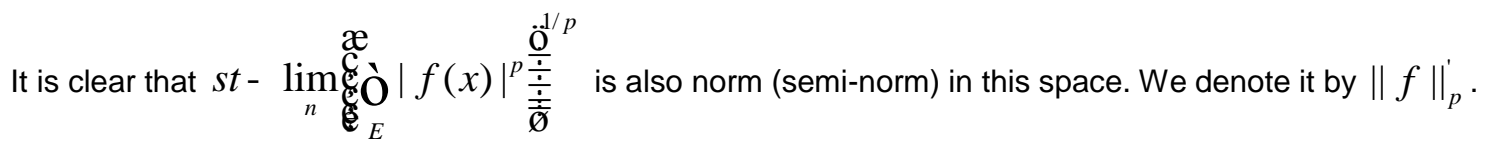

We prove that $L_{p}(\mu, X) \subset L^{\prime}{ }_{p}(\mu, X)$ in same way as is shown in [2] for $\mathrm{L}_{1}$ space.

Let $B$ be a sub $\sigma-$ field of $\Sigma$ and $f \in L_{p}^{\prime}(\mu, X)(1 \leq p<\infty)$. An element $g$ of $L_{p}^{\prime}(\mu, X)$ is called the stconditional expectation of $f$ relative to $B$ if $g$ is $B$ - measurable and

$$
s t-\int_{E} g d \mu=s t-\int_{E} f d \mu \text { for all } E \in B .
$$

In this case $g$ is denoted by $E(f \mid B)$.

Backward Martingale Let $\mathcal{F}_{n}$ be increasing sequence of $\sigma$-algebra, $n \leq 0$ such that

$\ldots \subset \mathcal{F}_{-3} \subset \mathcal{F}_{-2} \subset \mathcal{F}_{-1} \subset \mathcal{F}_{0}$. Let $f_{n}$ be $\mathcal{F}_{n}$ - adapted $n \leq 0$, and $E\left(f_{n+1} \mid \mathcal{F}_{n}\right)=f_{n}$.

\section{Convergence theorems of martingales}

In the following theorems we are extending in case of statistical convergence some results present in [18] on Banach space.

Theorem 1: Let $\left(f_{n}, \mathcal{F}_{n}, n \geq 1\right)$ be a martingale of st-Bochnerintegrable functions such that $f_{n}=E\left(f \mid \mathcal{F}_{n}\right), n \geq 1$ , where $f \in L_{p}\left(\Omega, \mathcal{F}_{\infty}, \mu\right), 1 \leq p<\infty$. Then

$$
s t-\lim _{n}\left\|f_{n}-f_{\infty}\right\|_{p}^{\prime}=0
$$

where $f_{\infty}=E\left(f \mid \mathcal{F}_{\infty}\right)$ and $\mathcal{F}_{\infty}=$ Borel - field generated by $\bigcup_{n} \mathcal{F}_{n}$.

Proof. Let $\mathcal{F}_{0}=\bigcup_{n=1}^{\infty} \mathcal{F}_{n}$. Clearly $\mathcal{F}_{\mathrm{o}}$ is a field. For $f \in L_{p}\left(\Omega, \mathcal{F}_{\infty}, \mu\right), 1 \leq p<\infty$, let 


$$
T_{n} f=E\left(f \mid \mathcal{F}_{n}\right)
$$

$\left\{T_{n}, n \geq 1\right\}$ is a sequence of bounded linear operators on the Banach space $L_{p}^{\prime}\left(\Omega, \mathcal{F}_{\infty}, \mu\right)$. If $f(\omega)=\chi_{F}(\omega) a$ where $a \in X, F \in \mathcal{F}_{0}$. Then since for some $N, F \in \mathcal{F}_{N}$ it follows that

$$
T_{n} f=f, \quad n \geq N
$$

Hence

$$
s t-\lim _{n}\left\|T_{n} f-f\right\|_{p}^{\prime}=0 .
$$

Therefore (1) is valid for all simple functions $f$ measurable with respect to $\mathcal{F}_{0}$. Since such functions are dense in $L_{p}\left(\Omega, \mathcal{F}_{\infty}, \mu\right)$ and $\left\|T_{n}\right\|=1$, the inequality

$$
\begin{aligned}
\left\|T_{n} f-f\right\|_{p}^{\prime} & \leq\left\|T_{n} g-g\right\|_{p}^{\prime}+\left\|T_{n} f-T_{n} g\right\|_{p}^{\prime}+\|g-f\|_{p}^{\prime} \\
& \leq\left\|T_{n} g-g\right\|_{p}^{\prime}+2\|g-f\|_{p}^{\prime}
\end{aligned}
$$

immediately establishes the validity of (1) for any $f \in L_{p}\left(\Omega, \mathcal{F}_{\infty}, \mu\right)$. Now for any $f \in L_{p}\left(\Omega, \mathcal{F}_{\infty}, \mu\right)$, $E\left(f \mid \mathcal{F}_{\infty}\right) \in L_{p}\left(\Omega, \mathcal{F}_{\infty}, \mu\right)$ and $T_{n} f=T_{n} E\left(f \mid \mathcal{F}_{\infty}\right)$. This along with the previous argument proves Theorem 1.

Theorem 2: Let $\left(f_{n}, \mathcal{F}_{n}, n \leq-1\right)$ be a martingale of st-Bochnerintegrable functions, let $f_{-1} \in L_{p}(\Omega, \Sigma, \mu)$, $1 \leq p<\infty$. Then

$$
s t-\lim _{n}\left\|f_{-\infty}-f_{-n}\right\|_{p}^{\prime}=0
$$

where $f_{-\infty}=E\left(f_{-1} \mid \mathcal{F}_{-\infty}\right)$ and $\mathcal{F}_{-\infty}=\bigcap_{n} \mathcal{F}_{-n}$.

Proof. The proof of Theorem 2 is similar to Theorem 1. One defines $T_{n} f=E\left(f \mid \mathcal{F}_{-n}\right)$ for $n \geq 1$, and $f \in L_{p}\left(\Omega, \mathcal{F}_{-1}, \mu\right)$. Using a classical martingale convergence theorem one proves the assertion in Theorem 2 for $f$ of the form $\chi_{F}(\omega) a$ where $F \in \mathcal{F}_{-1}$. The proof is then completed as in Theorem 1.

Lemma 1: Let $T_{n}, n \geq 1$ and T be bounded linear operators mapping the Banach space $\mathcal{Y}$ into itself and such that

i) $\quad s t-\lim _{n \rightarrow \infty} T_{n} f=T f$ for all $f \in \mathcal{Y}$ and

ii) $\quad T_{m} T_{n}=T_{\min (m, n)}$

Let $f_{n} \in \mathcal{Y}$ be such that $T_{n} f_{n+1}=f_{n}$ and that there is a subsequence $f_{n_{k}}$ statistical convergent weakly to $f_{\infty}$. Then $s t-\lim _{n \rightarrow \infty} f_{n}=f_{\infty}$.

Proof. From the conditions of the lemma, we have that

$$
T_{m} f_{n}=f_{\min (m, n)}(1)
$$

Also $T_{m} f_{n_{k}} \rightarrow T_{m} f_{\infty}$ as $k \rightarrow \infty$ for any $m$. For large $k$ because of (1), $T_{m} f_{n_{k}}=f_{m}$ and hence $T_{m} f_{\infty}=f_{m}$. By condition (i) of the lemma $s t-\lim _{m \rightarrow \infty} T_{m} f_{\infty}=T f_{\infty}$ so it follows that $s t-\lim _{m \rightarrow \infty} f_{m}=f_{\infty}$. 
Theorem 3: Let $\mathrm{X}$ be a reflexive Banach space and let $\left(f_{n}, \mathcal{F}_{n}, n \geq 1\right)$ be a martingale of $\mathrm{X}$-valued statistical Bochnerintegrable functions such that $f_{n} \in L_{p}\left(\Omega, \mathcal{F}_{\infty}, \mu\right), n \geq 1, \quad 1<p<\infty$ and $\left\|f_{n}\right\|_{p}^{\prime}<C$. Then there exists $f_{\infty} \in L_{p}(\Omega, \Sigma, \mu)$ such that $s t-\lim _{n}\left\|f_{n}-f_{\infty}\right\|_{p}^{\prime}=0$ and $f_{n}=E\left(f_{\infty} \mid \mathcal{F}_{n}\right)$.

\section{Proof.}

Let $\mathcal{F}_{\infty}=$ Borel - field generated by $\bigcup_{n} \mathcal{F}_{n}, T_{n} f=E\left(f \mid \mathcal{F}_{n}\right), \quad n \geq 1$ for $f \in L_{p}\left(\Omega, \mathcal{F}_{\infty}, \mu\right)$.

First consider the case $1<p<\infty . X$ being reflexive, $L_{p}\left(\Omega, \mathcal{F}_{\infty}, \mu, X\right)$ is also reflexive and hence the bounded set $\left\{f_{n}\right\}$ has a subsequence converging weakly to an element $f_{\infty}$ of $L_{p}\left(\Omega, \mathcal{F}_{\infty}, \mu, X\right)$. Thus in the case $1<p<\infty, T_{n}, n \geq 1$, I the identity operator, $I f=f$ and $f_{n}, n \geq 1$ satisfy all the conditions of lemma 1 . (To verify this one uses the fact that $\left(f_{n}, \mathcal{F}_{n}, n \geq 1\right)$ is a martingale and that Theorem 1 holds.) Hence the statement in Theorem 3 for $1<p<\infty$ follows immediately from lemma 1.

The case $p=1$ is dealt with exactly similarly.

\section{References}

[1] H. Çakalli, A study on statistical convergence. Functional analysis, approximation and computation 1:2(2009), 19-24.

[2] A. Caushi, A. Tato, A statistical integral of Bochner type on Banach space, Hikari Ltd Appl. Math. Sci., Vol. 6, 2012 , no. 137-140, 6857-6870.

[3] J. Connor, M. Ganichev and V. Kadets, "A characterization of Banach spaces with separable duals via weak statistical convergence," Journal of Mathematical Analysis and Applications, vol. 244, no. 1, pp. 251-261, 1989.

[4] J. Diestel and J. J. Jr. Uhl,"Vector measure”, Math. Surveys no.15. Providence (1977).

[5] J.A. Fridy, "On statistical convergence," Analysis, vol. 5, no. 4, pp. 301-313, 1985.http://dx.doi.org/10.1524/anly.1985.5.4.301

[6] J.A. Fridy, "Statistical limit points," Proceedings of the American Mathematical Society, vol. 118, no. 4, pp. 1187-1192, 1993.

http://dx.doi.org/10.2307/2160076

[7] J.A. Fridy, C. Orhan, Statistical limit superior and limit inferior, Proc. Amer. Math. Soc., 125, nr. 12(1997) 3625-3631.

http://dx.doi.org/10.1090/s0002-9939-97-04000-8

[8] A.D. Gadijev and C. Orhan, Some approximation theorems via statistical convergence, Rocky Mountain j. Math., 32 (2002), 129-138.

[9] A. Gökhan, M. Güngör, Onpointwise statistical convergence, Indian Journal of pure and application mathematics, 33(9): 1379-1384, 2002.

[10] H. I. Miller, A measure theoretical sub sequence characterization of statistical convergence. Trans. Amer. Math. Soc., 347, 1819-1881, 1995.

http://dx.doi.org/10.1090/s0002-9947-1995-1260176-6

[11] I. J. Schoenberg, The integrability of certain functions and related summability methods. The American Mathematical Monthly, vol 66, no. 5 pp. 361-375, 1959. http://dx.doi.org/10.2307/2308747

[12] A. Zigmund, Trigonometric Series. Second sd., Cambridge Univ. Press, Cambridge, 1979.

[13] Fast, H. (1951), Sur la convergence statistiques, colloq. Mathematics 2:241-244.

[14] T. Salat, "On statistically convergent sequences of real numbers," Math. Slovaca, 30 (1980), 139-150.

[15] J. Fridy, C. Orhan, "Lacunary statistical summability," J. Math. Anal. Appl. Vol.173, pp. 497-504, 1993.

[16]D. Braho, E. Donefski, "On the martingale statistical convergence and Radon-Nikodym property," Global Journal of Mathematics, Vol. 6, No. 3, pp. 630-635, 2016.

[17]D. Braho, A. Caushi, "On the Statistical Convergence of Martingales," Hikari Ltd Appl. Math. Sci., Vol. 9, no. 17, 821830, 2015.

[18]S. D. Chatterji, A note on the convergence of Banach-space valued martingales, Math. Ann. 153 (1964), pp. $142-149$. 